\title{
Piezotronic effect in a normally off p-GaN/ AlGaN/GaN HEMT toward highly sensitive pressure sensor
}

Cite as: Appl. Phys. Lett. 118, 242104 (2021); https://doi.org/10.1063/5.0053701

Submitted: 09 April 2021 . Accepted: 26 May 2021 . Published Online: 17 June 2021

Hong-Quan Nguyen, (i) Thanh Nguyen, Philip Tanner, Tuan-Khoa Nguyen, Abu Riduan Md Foisal, Jarred FastierWooller, Tuan-Hung Nguyen, (D) Hoang-Phuong Phan, (D) Nam-Trung Nguyen, and Dzung Viet Dao

ARTICLES YOU MAY BE INTERESTED IN

Demonstration of $\mathrm{AlGaN} / \mathrm{GaN}$-based ultraviolet phototransistor with a record high

responsivity over $3.6 \times 10^{7} \mathrm{~A} / \mathrm{W}$

Applied Physics Letters 118, 242105 (2021); https://doi.org/10.1063/5.0055468

Electron overflow of AIGaN deep ultraviolet light emitting diodes

Applied Physics Letters 118, 241109 (2021); https://doi.org/10.1063/5.0055326

Reverse leakage and breakdown mechanisms of vertical GaN-on-Si Schottky barrier diodes with and without implanted termination

Applied Physics Letters 118, 243501 (2021); https://doi.org/10.1063/5.0049706

母QBLOX

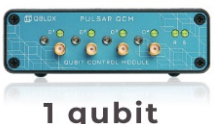

Shorten Setup Time Auto-Calibration More Qubits

Fully-integrated Quantum Control Stacks Ultrastable DC to $18.5 \mathrm{GHz}$ Synchronized $<<1$ ns Ultralow noise

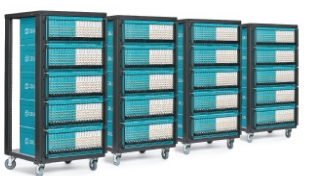

100s qubits

visit our website > 


\title{
Piezotronic effect in a normally off p-GaN/AIGaN/ GaN HEMT toward highly sensitive pressure sensor
}

Cite as: Appl. Phys. Lett. 118, 242104 (2021); doi: 10.1063/5.0053701

Submitted: 9 April 2021 - Accepted: 26 May 2021 .

Published Online: 17 June 2021

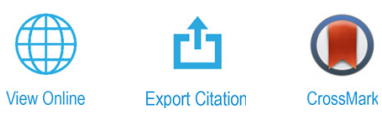

Hong-Quan Nguyen,, (1) Thanh Nguyen, ${ }^{1}$ (iD Philip Tanner, Tuan-Khoa Nguyen, ${ }^{\top}$ Abu Riduan Md Foisal, Jarred Fastier-Wooller,' Tuan-Hung Nguyen, Hoang-Phuong Phan, (iD Nam-Trung Nguyen, and Dzung Viet Dao

\begin{abstract}
AFFILIATIONS
${ }^{\top}$ Queensland Micro and Nanotechnology Centre, Griffith University, Queensland, Australia

${ }^{2}$ School of Engineering and Built Environment, Griffith University, Queensland, Australia
\end{abstract}

a) Author to whom correspondence should be addressed: quan.nguyen@griffith.edu.au

\begin{abstract}
We report the effect of stress or strain on the electronic characteristics of a normally off AlGaN/GaN high electron mobility transistor (HEMT) and demonstrate its role as a highly sensitive pressure sensor. We observe that the HEMT drain current exhibits a linear change of $2.5 \% /$ bar upon the application of pressure, which is translated to a strain sensitivity of $1250 \mathrm{ppm}^{-1}$. This is the highest strain sensitivity ever reported on HEMTs and many other conventional strain sensing configurations. The relative change of drain current is largest when the gate bias is near-threshold and drain bias is slightly larger than the saturation bias. The electron sheet density and mobility changes in the AlGaN/GaN heterointerface under the applied pressure or mechanical strain are explained qualitatively. The spontaneous and piezoelectricpolarization-induced surface and interface charges in the AlGaN/GaN heterojunction can be used to develop very sensitive and robust pressure sensors. The results demonstrate a considerable potential of normally off AlGaN/GaN HEMTs for highly sensitive and reliable mechanical sensing applications with low energy consumption.
\end{abstract}

Published under an exclusive license by AIP Publishing. https://doi.org/10.1063/5.0053701

In recent years, gallium nitride $(\mathrm{GaN})$ has become a popular semiconductor material for advanced electronic and power switching devices. Compared to silicon (Si), GaN has several superior properties such as widebandgap, high critical breakdown electric field, high thermal conductivity, and high electronic saturation velocity for power electronics. ${ }^{1,2}$ Moreover, GaN-based materials possess many advantages such as high biocompatibility, ${ }^{3}$ high thermal and mechanical stability, ${ }^{4-6}$ and excellent irradiation resistance, ${ }^{7}$ which are critical for applications in harsh environments. ${ }^{8}$ Another unique property of GaN-based materials is the possibility of bandgap engineering by tailoring the strain effect in GaN-related alloys and heterostructures. In particular, a two-dimensional electron gas (2DEG) with a high sheet carrier density and mobility is generated at the AlGaN/GaN heterojunction, owing to the spontaneous and piezoelectric polarization, and the AlGaN surface state acting as an electron source. The spontaneous polarization (PSP) is caused by the asymmetry of the wurtzite crystal structure of III-nitrides, while the piezoelectric polarization (PPE) originates from the strained $\mathrm{AlGaN}$ barrier layer due to lattice mismatch between $\mathrm{AlGaN}$ and $\mathrm{GaN} .{ }^{10-12}$ Thanks to the strong PPE effects, obvious changes in the conductivity of the 2DEG channel can be seen when applying external stress onto an $\mathrm{AlGaN} / \mathrm{GaN}$ heterostructure, which promises applications in stress/strain sensors ${ }^{13-16}$ and pressure sensors. $^{17-21}$

$\mathrm{AlGaN} / \mathrm{GaN}$ pressure sensors based on simple piezoresistors and gated piezoresistors have been reported earlier, but they suffer from either low sensitivity or a complex fabrication process. ${ }^{22,23}$ Recently, the use of a high electron mobility transistor (HEMT) as the sensing element has led to a significant increase in sensitivity along with potential for the integration of sensors with conditioning circuits. ${ }^{12,1}$

Generally, high electron mobility transistors (HEMTs) are in the normally on (depletion-mode or d-mode) state, with typical threshold voltages to switch off devices ranging from -6 to $-2 \mathrm{~V}$. Compared to normally on counterparts, normally off (depletion-mode or D-mode) HEMTs have attracted significant attention due to significant reduction in power consumption during the standby state and enhanced reliability. Moreover, the configuration of normally off HEMT devices allows for simpler power amplifier circuits and high-power switches by eliminating the requirement for negative-polarity voltage supply, thus significantly reduces the circuit complexity and system cost. ${ }^{24}$ Among technologies such as recessed gate and fluorine plasma 
implantation, ${ }^{25-27}$ the $\mathrm{p}-\mathrm{GaN}$ gate on $\mathrm{AlGaN} / \mathrm{GaN}$ is the only commercially available solution for normally off HEMTs with good device performance and reliability. ${ }^{28}$

While to date, normally on HEMTs have been investigated for pressure sensing application, ${ }^{8,29-32}$ there has been no report on the dependence of electrical properties of normally off AlGaN/GaN HEMT on external mechanical stimuli. This paper aims to investigate the influence of pressure on the characteristics of p-Gan/AlGaN/GaN HEMT device. The insights achieved in this study are important, not only for designing micro-electro-mechanical systems' (MEMS) mechanical sensors but also significant for understanding the behaviors of GaN-based devices, which can be affected by strain due to packaging processes as well as in applications.

In this paper, we implemented a pressure sensor with a $\mathrm{p}-\mathrm{GaN} /$ $\mathrm{AlGaN} / \mathrm{GaN}$ heterojunction as the sensing element with the sensor configuration shown in Fig. 1. The sensing element was located in the middle of the diaphragm, and the applied pressure was detected via the changes in drain current $\Delta I_{D S}$ under optimal magnitudes of the gate voltage $V_{G S}$ and the drain voltage $V_{D S}$.

A p-GaN/AlGaN/GaN heterojunction was grown by a metal organic chemical vapor deposition (MOCVD) technique on a $750 \mu \mathrm{m}$ thick 6 in. Si (111) wafer. The structure consists of a $100 \mathrm{~nm} \mathrm{p-GaN}$ layer with a $\mathrm{Mg}$ doping concentration of $3 \times 10^{19} \mathrm{~cm}^{-3}$, an $18 \mathrm{~nm}$ undoped $\mathrm{AlGaN}$ barrier, and a $1.5 \mu \mathrm{m} \mathrm{GaN}$ channel layer. In a similar HEMT structure fabricated without the $\mathrm{p}-\mathrm{GaN}$ gate, the 2DEG electron density and electron mobility were measured to be $9.3 \times 10^{13} \mathrm{~cm}^{-2}$ and $2150 \mathrm{~cm}^{2} / \mathrm{V} \mathrm{s}$, respectively, resulting in a sheet resistance of $310 \Omega / \square$.

The device fabrication started with patterning of the $\mathrm{p}-\mathrm{GaN}$ to form gate areas using inductively coupled plasma (ICP) etching. This was followed by the source and drain Ohmic contact formation that consisted of $\mathrm{Ni} / \mathrm{Ti}(20 / 100 \mathrm{~nm})$ sputter deposition, patterning and rapid thermal annealing at $800^{\circ} \mathrm{C}$ for $2 \mathrm{~min}$ in $\mathrm{N}_{2}$ ambient. $\mathrm{Ni}$ was then deposited and patterned to create top metal contacts for the gate, source, and drain. Due to the circular shape of the device with the grounded source contact as the outer ring, no device-to-device isolation was required. Subsequently, the devices were passivated with $150 \mathrm{~nm}$ SiN using a plasma enhanced chemical vapor deposition (PECVD) process. The device has a gate length $L_{g}$ of 250 and a width
$W_{g}$ of $2455 \mathrm{~nm}$. The spacings of the gate-drain $L_{g d}$ and gate-source $L_{g s}$ were the same at $16 \mathrm{~nm}$. Deep reactive ion etching (DRIE) was employed to achieve a circular diaphragm by thinning down the silicon substrate to $150 \mu \mathrm{m}$ from the back side. The total etching depth was approximately $600 \mu \mathrm{m}$, and the diameter of the diaphragm was $300 \mu \mathrm{m}$. The final step is dicing the wafer into smaller sensor chips. The detailed fabrication process is shown in supplementary material Fig. S1. The sensor was then attached to a chip holder with an open window at the center using an adhesive epoxy, as depicted in Fig. S2(b). The inset of Fig. S2(b) shows a completed sensor with bonding wires.

Supplementary material Fig. S2 shows the schematic and experimental setup to investigate the performance of the pressure sensor at room temperature. Air pressure was applied to the back side of the diaphragm using an ELVEFLOW OB1 pressure controller combined with a pneumatic pump. A B1500A semiconductor device analyzer was used to control the tuning voltages and simultaneously measure the drain current. The transfer and output characteristics of the normally off AlGaN/GaN HEMTs were also measured using the B1500A semiconductor device analyzer. Results and further discussion can be found in the supplementary material, Sec. II.

The impact of the membrane pressure on the charge transport in a normally off HEMT device was evaluated using DC electrical measurements, Fig. 2. Figure 2(a) plots the $I_{D S^{-}} V_{D S}$ response of the sensor under pressures from 0 to $1.6 \mathrm{bar}$ at room temperature with the gate voltage $V_{G S}$ varying from 0 to $0.9 \mathrm{~V}$. Figures 2(b) and 2(c) show the $I_{D S^{-}} V_{D S}$ characteristics of the sensor in unsaturated and saturated drain current regions, respectively. In both regions, the drain current $I_{D S}$ decreases with the increasing pressure. This reduction will be explained by the energy band diagram in the upcoming section. The pressure also has a significant impact on both the threshold voltage and transconductance, as discussed in the supplementary material, section 3 .

The influence of drain voltage $V_{D S}$ on the relative change of the drain current $\Delta I_{D S} / I_{o}$ was investigated at the specific gate voltage of $V_{G S}=0.7 \mathrm{~V}$, Figure 3 (a). $\Delta I_{D S} / I_{o}$ undergoes a large increase from $0 \%$ to $1.6 \%$ as $V_{D S}$ increases from 0 to $0.4 \mathrm{~V}$. With further increase in $V_{D S}$ to $3 \mathrm{~V}$, the relative change plateaus and then varies around the saturation level. For $V_{D S}=0.5 \mathrm{~V}$, the response $\Delta I_{D S} / I_{o}$ was $1.6 \pm 0.01 \%$. At (a) Diaphram $(\varnothing 3000 \mu \mathrm{m}, \mathrm{t}=150 \mu \mathrm{m})$

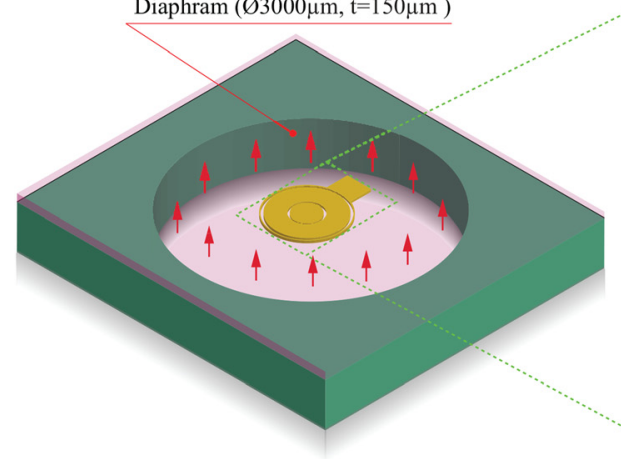

(b)

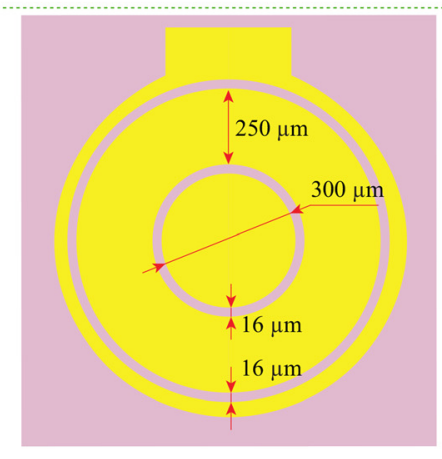

(c)

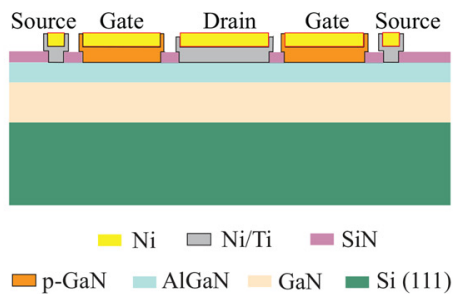

FIG. 1. Schematic view of the normally off p-GaN/AIGaN/GaN HEMT pressure sensor. (a) 3D schematic of the sensor. (b) Top view of the p-GaN/AIGaN/GaN HEMT sensing element. (c) Cross-sectional view of the AIGaN/GaN/Si diaphragm with the thickness $t=150 \mu \mathrm{m}$. 
(a)

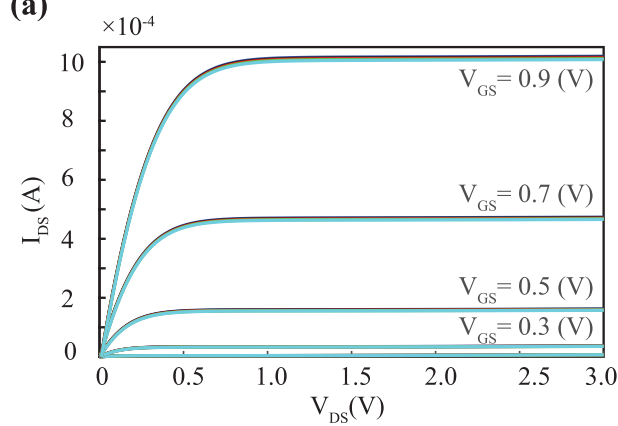

(b)

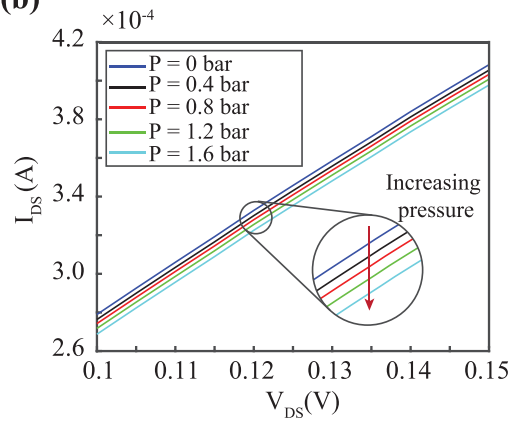

(c)

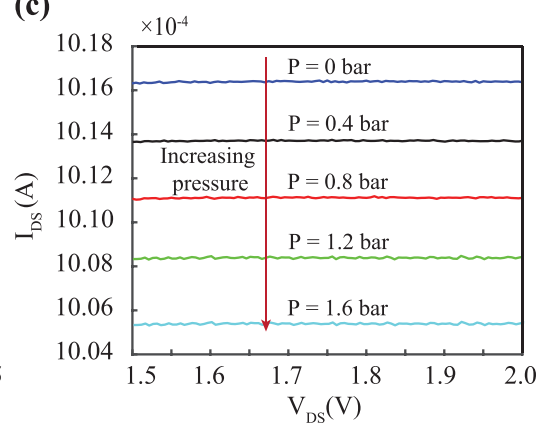

FIG. 2. Impact of pressure on the output characteristics of the normally off HEMT sensing device. (a) Drain current $I_{D S}$ vs drain voltage $V_{D S}$ at a pressure range of $0-1.6$ bar under the gate voltage $V_{G S}$ in the range of $0-0.9 \mathrm{~V}$. (b) $I_{D S} v S V_{D S}$ at the pressure range of $0-1.6$ bar under $V_{G S}=0.9 \mathrm{~V}$ and the unsaturated region $V_{D S}$ ranging from 0.1 to $0.15 \mathrm{~V}$. (c) $I_{D S}$ VS $V_{D S}$ at the pressure range of $0-1.6$ bar and $V_{G S}=0.9 \mathrm{~V}$ at the saturated region $V_{D S}$ ranging from 1 to $1.5 \mathrm{~V}$.

higher $V_{D S}$, noise in $I_{D S}$ increased up to $\pm 0.1 \%$ at $V_{D S}=2.5 \mathrm{~V}$, i.e., approximately ten times larger than that at $V_{D S}=0.5 \mathrm{~V}$. Possible explanations for the dispersion in the response at higher $V_{D S}$ include hot carrier injection into traps in the gate barrier layer, surface states, and self-heating. Similar trends for AlGaN/GaN HEMTs were previously reported. ${ }^{33,34}$ For instance, Le Boulbar et al. reported that the sensitivity of AlGaN/GaN HEMTs increases with increasing $V_{D S}$, until it saturated.

Figure 3(b) presents the maximum relative change in drain current $\left(\Delta I_{D S, s a t} / I_{D S, o}\right)$ as a function of pressure for different gate voltages. It can be observed that the maximum relative change in drain current follows a good linear relationship with the applied pressure from 0 to $1.6 \mathrm{bar}$ at applied gate voltages from 0.3 to $0.9 \mathrm{~V}$. The largest relative change occurs at a gate bias $V_{G S}$ near $V_{T h}=0.3 \mathrm{~V}$ and a drain bias $V_{D S}$ near $0.5 \mathrm{~V}$. Along with good linearity of the output signal, the device possesses excellent repeatability with cyclic pressure, as shown in Fig. S4. The experimental data exhibit good reproducibility of the output signal with high signalto-noise ratio, Fig. S4(b). follows:

Pressure sensitivity $S_{P}$ and strain sensitivity $S_{\varepsilon}$ are defined as

$$
S_{P}=\frac{\Delta I_{D S}}{I_{D S, o}} \frac{1}{P}
$$

$$
S_{\varepsilon}=\frac{\Delta I_{D S}}{I_{D S, o}} \frac{1}{\varepsilon},
$$

where $\Delta I_{D S}=I_{D S}-I_{D S}{ }_{o}$ is the change in current across the sensing element due to applied pressure, $I_{D S}$, is the current across the sensing element under a pressure-free condition, $P$ is the applied pressure, and $\varepsilon$ is the strain induced in the HEMT structure. Finite element analysis using Comsol Multiphysics ${ }^{\mathrm{TM}}$ was employed to estimate the strain induced in the sensing area where the HEMT is located.

Figure 3(c) plots the pressure sensitivity calculated using linear regression of the maximum relative change in current against the applied pressure. The pressure sensitivity was approximately $0.1 \% / \mathrm{bar}$ at the gate voltage of $V_{G S}=1.5 \mathrm{~V}$, and this sensitivity significantly increases by 25 times, i.e., up to $2.5 \% / \mathrm{bar}$ at $V_{G S}=0.3 \mathrm{~V}$. This large disparity in sensitivity might be due to the pinched-off effect of the channel. This can be explained by the fact that the current decreases at lower positive gate voltages; however, the applied piezoelectric polarization from pressure remains the same regardless of the gate voltage. Thus, a change in gate voltage leads to a higher change in current with respect to pressure, because of the reduction in the overall current. The drain current exhibits a linear change of $2.5 \%$ /bar upon the application of pressure, which is equivalent to a strain sensitivity $S_{\varepsilon}$ of $1250 \mathrm{ppm}^{-1}$. This is the highest strain sensitivity ever reported on (a)

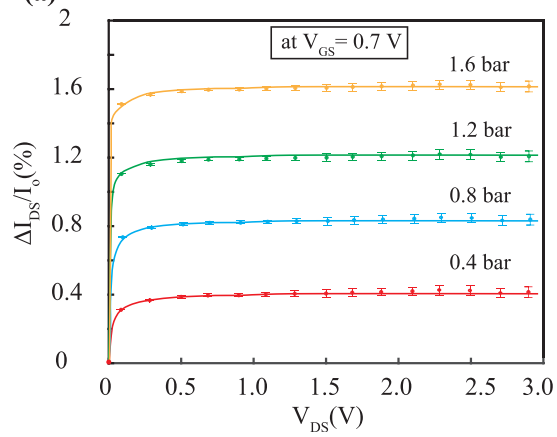

(b)

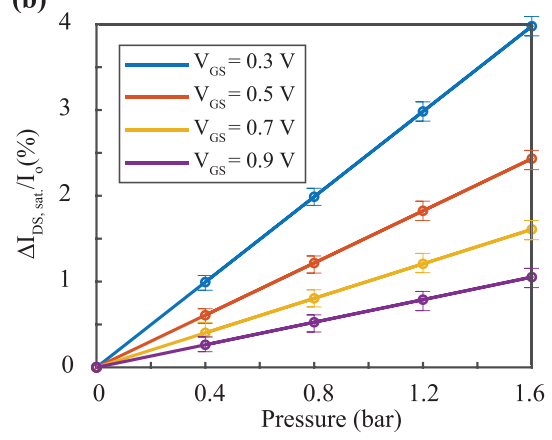

(c)

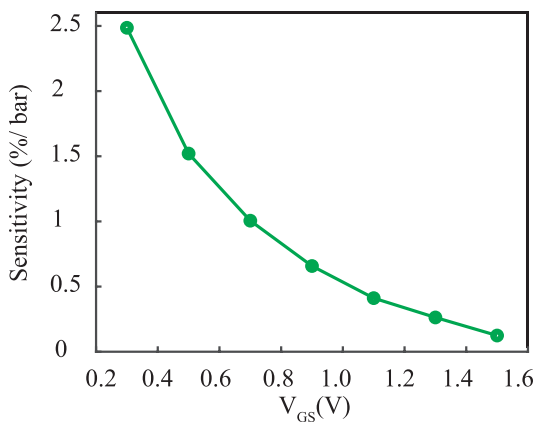

FIG. 3. Pressure sensitivity at different drain voltage and gate voltage. (a) Relative change in drain current vs drain voltage for pressures from 0.4 to 1.6 bar. (b) Maximum relative change in $I_{D S} v s$ pressure at different gate voltages $V_{G S}$. (c) Device sensitivity as a function of $V_{G S}$ determined by linear regression of maximum $\Delta I_{d} I_{0} v s$ pressure. 
HEMT and many other conventional strain sensing configurations. The detailed comparison can be found in Sec. VI of the supplementary material.

To further understand the relationship between the electronic properties of HEMT and the applied pressure/strain, we investigate the variation of the polarization charges and mobility under application of pressure/strain.

Under stress-free conditions, there are spontaneous polarizations $P_{S P}^{A l G a N}$ and $P_{S P}^{G a N}$ due to the non-coincidence of positive and negative charge centers of $\mathrm{AlGaN}$ and $\mathrm{GaN}$, along with piezoelectric polarization $P_{l m}^{A l G a N}$ due to the lattice mismatch between AlGaN and GaN layers.

Lattice-mismatch induced piezoelectric polarization $P_{l m}^{A l G a N}$ in $\mathrm{AlGaN}$ can be given as ${ }^{10}$

$$
P_{l m}^{A l G a N}=2 e_{31}^{A l G a N} \epsilon_{1}^{A l G a N}+e_{33}^{A l G a N} \epsilon_{3}^{A l G a N},
$$

in which $e_{31}^{A l g a N}$ and $e_{33}^{A l G a N}$ are the piezoelectric constants, and $\epsilon_{1}^{A l G a N}$ and $\epsilon_{3}^{A l G a N}$ are the components of the strain field

$$
\begin{gathered}
\epsilon_{1}^{A l G a N}=\frac{a^{G a N}-a^{A l G a N}}{a^{A l G a N}}, \\
\epsilon_{3}^{A l G a N}=-2 \frac{c_{11}^{A l G a N}}{c_{33}^{A l G a N}},
\end{gathered}
$$

where $a^{A l G a N}$ and $a^{G a N}$ are the lattice constants of the AlGaN and $\mathrm{GaN}$, respectively. $c_{11}^{A l G a N}$ and $c_{33}^{\text {AlGaN }}$ are the elastic stiffness constants of AlGaN.

The elastic stiffness constants $c_{33}^{A l G a N}$ and $c_{11}^{A l G a N}$ and piezoelectric constants of $\mathrm{AlGaN} e_{31}^{\text {AlgaN }}$ and $e_{33}^{\text {AlgaN }}$ can be calculated based on Vegard's law and data of AlN and GaN from previous research ${ }^{12,35}$

$$
\begin{aligned}
& e_{31}^{A l G a N}=x e_{31}^{A l N}+(1-x) e_{31}^{G a N} ; e_{33}^{A l G a N}=x e_{33}^{A l N}+(1-x) e_{33}^{G a N}, \\
& c_{11}^{A l G a N}=x c_{11}^{A l N}+(1-x) c_{11}^{G a N} ; c_{33}^{A l G a N}=x c_{33}^{A l N}+(1-x) c_{33}^{G a N} .
\end{aligned}
$$

In the meantime, the spontaneous polarization in $\mathrm{AlGaN}$ can also be estimated from

$$
P_{s p}^{A l G a N}=x P_{s p}^{A l N}+(1-x) P_{s p}^{G a N}
$$

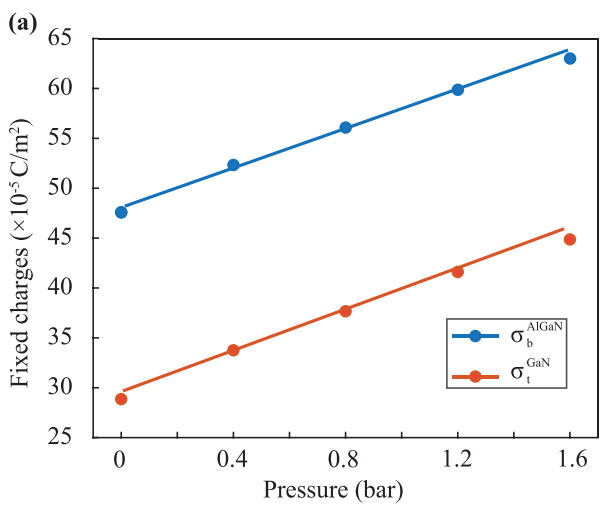

$\mathrm{Al}$ composition in the $\mathrm{AlGaN}$ layer is characterized and estimated to be $\sim 25 \%$ thus $\mathrm{x}=0.25$. Furthermore, the spontaneous polarizations in $\mathrm{AlN}$ and $\mathrm{GaN}$ have been given in previous report with $P_{s p}^{A l N}=-0.081$ and $P_{s p}^{G a N}=-0.029 \mathrm{C} / \mathrm{m}^{2} .{ }^{36}$

The strain-induced piezoelectric polarization in AlGaN and GaN can be obtained by ${ }^{22}$

$$
\begin{aligned}
P_{p z}^{A l G a N} & =-\frac{F_{b}^{A l G a N}}{K_{A l G a N}}, \\
P_{p z}^{G a N} & =-\frac{F_{b}^{G a N}}{K_{G a N}},
\end{aligned}
$$

in which $K_{A l G a N}$ and $K_{G a N}$ are the dielectric constants of AlGaN and $\mathrm{GaN}$, respectively.

The strain-induced electric fields at the bottom of AlGaN layer $\left(F_{b}^{A l G a N}\right)$ and that at the top of GaN $\left(F_{t}^{G a N}\right)$ are simulated by FEA software COMSOL Multiphysics. The p-GaN/AlGaN/GaN/Si heterostructure is modeled as a cylinder with a radius of $3000 \mu \mathrm{m}$ and a height of $150 \mu \mathrm{m}$. The material constants of $\mathrm{AlGaN}, \mathrm{GaN}$, and $\mathrm{Si}$, including the mass density, Young's modulus, the piezoelectric constants, and the dielectric constants used in the calculations, are obtained from previous research. ${ }^{35,37}$ A boundary displacement is defined by the pressure applied upward on the Si bottom layer with the side of the cylinder fixed.

The effective fixed polarization charges at the bottom of AlGaN and the absolute value of the effective fixed polarization charges at the top of $\mathrm{GaN}$ are ${ }^{12}$

$$
\begin{gathered}
\sigma_{b}^{A l G a N}=P_{l m}+P_{s p}^{A l G a N}-P_{p z}^{A l G a N}, \\
\left|\sigma_{t}^{G a N}\right|=\left|P_{s p}^{A l G a N}-P_{p z}^{A l G a N}\right|
\end{gathered}
$$

The net fixed polarization charges at the $\mathrm{AlGaN} / \mathrm{GaN} / \mathrm{GaN}$ heterostructure interface can be obtained as ${ }^{12}$

$$
\sigma^{i n t}=\sigma_{b}^{A l G a N}-\left|\sigma_{t}^{G a N}\right| .
$$

The calculated results under strain-free and tension strains are shown in Figs. 4(a) and 4(b). Free electrons close to the heterostructure interface are attracted by the net fixed polarization charges and

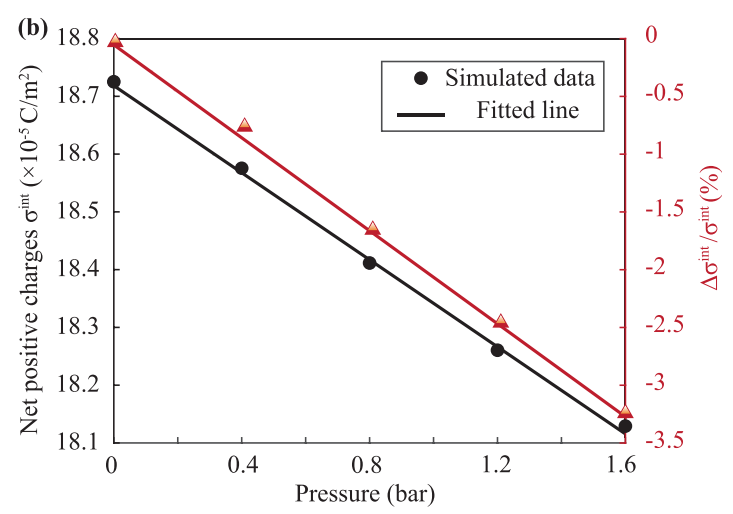

FIG. 4. Piezotronic effect and fixed charge distribution in the AIGaN/GaN heterostructure. (a) The fixed polarization charges distributed at the bottom of AIGaN layer and the absolute value of fixed polarization charges distributed at the top surface of $\mathrm{GaN}$ as a function of external pressure/strain applied. (b) The effective net fixed polarization charges at AIGaN/GaN heterojunctions vs applied pressure. 
(a)

\section{Strain Free with $V_{G S}=0 \mathrm{~V}$}
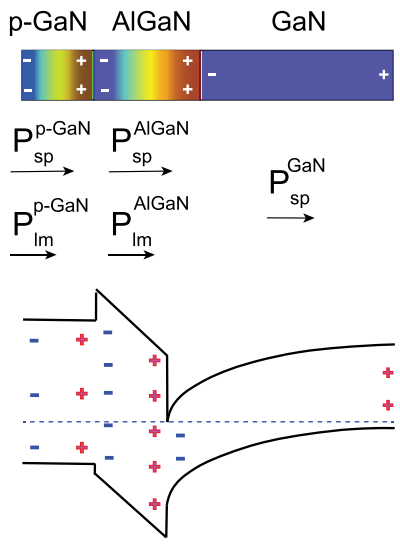

(b)

Strain Free with $V_{G s}>V_{T h}$
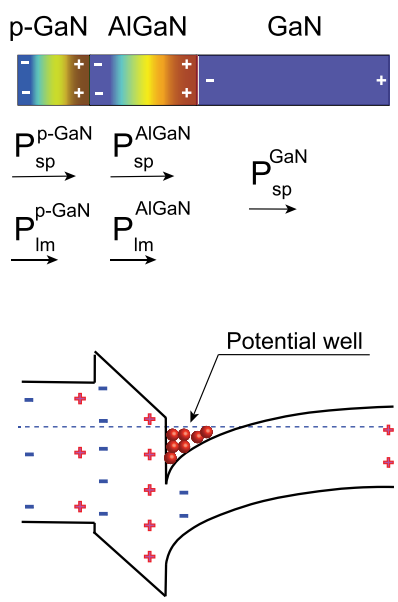

(c)

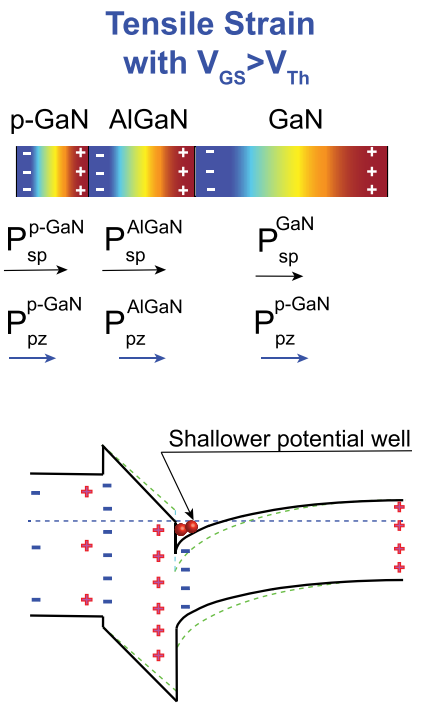

FIG. 5. Band diagrams of the $p-G a N / A I G a N / G a N$ heterojunction under (a) strain free condition with $V_{G S}=0 V$, (b) strain free condition and with $V_{G S}>V_{T h}$, and (c) under tensile strain condition.

confined in the potential well, giving rise to the formation of 2DEG under a stress-free condition, Fig. 5(b). As shown in Fig. 4(a), the tensile strain gives rise to the differentiation between $\mathrm{AlGaN}$ bottom surface's charges and the GaN top surface's charges, which cause the decline of net fixed polarization charges $\sigma^{\text {int }}$. The relative change of $\sigma^{\text {int }}$ is determined to be $-2.05 \% /$ bar, Fig. $4($ b) . Accordingly, a certain amount of the confined free electrons is released from the potential well and thus decreases the sheet density of 2DEG. In addition, the negative piezoelectric charge accumulates at the heterojunction interface, and raising the energy band on the GaN side and making the triangular potential well at the heterojunction interface become shallow, as shown in Fig. 5(c). The shallower potential well causes binding carriers to be weak, and the 2DEG concentration decreases. As external tensile stress increases, this modulation is also strengthened. The combination of reduced net fixed charge and shallower potential well leads to the decrease in 2DEG sheet density under tensile strain. The calculated result of sheet density shown in Fig. 6(a) agrees well with the proposed mechanism. The detailed calculation can be found in Sec. VI of the supplementary material.

To confirm the proposed physical mechanism and theoretical calculation, a Hall effect measurement was conducted on a four terminals device. The detailed setup can be found in section 6 of the supplementary material. From Fig. 6(a), we observed a decrease in the 2DEG sheet density in the $\mathrm{p}-\mathrm{GaN} / \mathrm{AlGaN} / \mathrm{GaN}$ heterostructure with an increase in pressure by $-2.46 \% / \mathrm{bar}$ at a gate voltage $V_{G S}$ of $0.3 \mathrm{~V}$.

The conductivity of the sensing element also depends on electron mobility. Electron mobility change is resulted from shifting of the average conductivity effective mass and intervalley/intravalley scattering. ${ }^{38}$ Stress affects electron effective mass through two factors: band warping and band-splitting induced electron repopulation. Unlike Si, which has six degenerate conduction band valleys or GaAs, which has energetically adjacent conduction bands, $\mathrm{GaN}$ is a direct bandgap material with only one conduction band minimum located at the $\Gamma$-point. ${ }^{39}$ Consequently, no electron repopulation occurs under
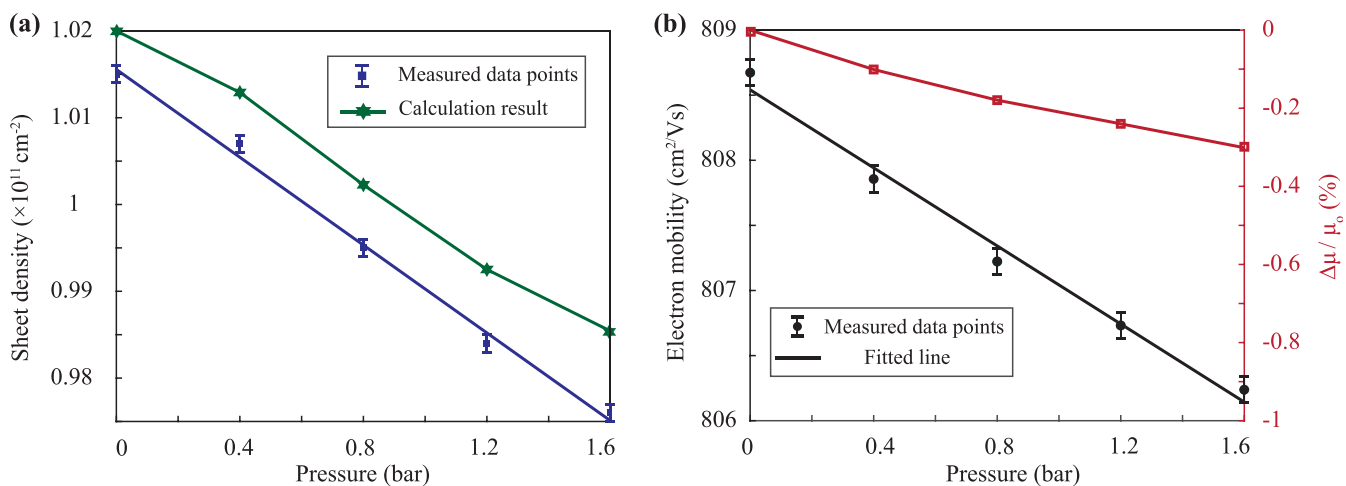

FIG. 6. (a) Self-consistent calculation result and measurement result of 2DEG sheet density $N_{S}$ and (b) measured mobility $\mu$ under different pressures. 
stress. The absence of stress-induced conduction band splitting also results in a negligible change in the intervalley scattering. Therefore, the band warping is hypothesized as the dominant mechanism for effective mass change with stress.

The effective mass $m^{*}$ can be presented as ${ }^{40}$

$$
m^{*}=\hbar^{2} \frac{1}{\frac{d_{E}^{2}}{d k^{2}}},
$$

where $\hbar$ is the Planck constant, $E$ is the energy, and $k$ is the wave number. We can see that the effective mass $m^{*}$ is inversely proportional to the energy band curvature $\frac{d_{E}^{2}}{d k^{2}}$.

If a tensile stress is applied to the $\mathrm{AlGaN} / \mathrm{GaN}$ heterostructure, the conduction band shifts to a lower energy level along with the reduction in the energy band curvature $\frac{d_{E}^{2}}{d k^{2}}$ as reported by Jogai ${ }^{41}$ and Postorino et al. ${ }^{42}$

Thus, the effective mass of the 2DEG increases with the application of tensile stress. This agrees well with the previous result from Chu et al. ${ }^{43}$ From the Drude model, it is known that the carrier mobility is inversely proportional to the charge carrier effective mass $\left(\mu \propto 1 / m^{*}\right){ }^{44}$ As a result, tensile strain causes the reduction in 2DEG mobility of the $\mathrm{p}-\mathrm{GaN} / \mathrm{AlGaN} / \mathrm{GaN}$ heterostructure. To confirm the theoretical assumption and determine the contribution of mobility to the piezotronic effect, mobility of the electron in 2DEG was measured and the result is shown in Fig. 6 (b). The decline trend of the mobility completely agrees with the hypothesis, and the relative change $\Delta \mu / \mu_{o}$ of the 2DEG with pressure was determined to be $-0.2 \% / b a r$, as shown in Fig. 6(b). This change in mobility is negligible compared with the variation of the sheet density under application of pressures; hence the polarization charge variation is the dominant mechanism that is responsible for the piezotronic effect in the $\mathrm{p}-\mathrm{GaN} / \mathrm{AlGaN} / \mathrm{GaN}$ heterostructure.

In conclusion, we report the influence of mechanical pressure on the electrical properties of normally off AlGaN/GaN HEMTs. Our experimental data indicate that the drain current of the normally off AlGaN/GaN HEMT decreases with the increasing pressure or tensile stress, where the change is most profound near the threshold voltage. We also observed that the bias and gate voltages play a significant role in the stress/strain sensitivity of the HEMT device. The sensitivity of the device has increased by almost $1000 \%$ by tuning the drain and gate voltages into the optimum values. Our findings demonstrated the potential of normally off AlGaN/GaN HEMTs for highly sensitive mechanical sensor applications.

See the supplementary material for the fabrication processes, characterization of piezotronics effect of the HEMT, transfer and output characteristics of the normally off HEMT, repeatability of the pressure sensor, comparison of sensitivities, and sheet density calculation and mobility measurement.

This project was partially supported by the Foundation for Australia-Japan Studies under the Rio Tinto Australia-Japan Collaboration Project and by Australian Research Council Grant No. LP160101553.

\section{DATA AVAILABILITY}

The data that support the findings of this study are available from the corresponding author upon reasonable request.

\section{REFERENCES}

${ }^{1}$ S. M. Sze, Physics of Semiconductor Devices (John Wiley \& Sons, 2012).

${ }^{2}$ M. A. Khan, G. Simin, M. S. Shur, R. Gaska, and G. K. Sujan, Reference Module Materials in Science and Materials Engineering (Elsevier, 2018), p. 1.

${ }^{3}$ M. S. Shur and R. F. Davis, GaN-Based Materials and Devices (World Scientific, 2004).

${ }^{4}$ N. I. Kim, Y. L. Chang, J. Chen, T. Barbee, W. Wang, J. Y. Kim, M. K. Kwon, S. Shervin, M. Moradnia, S. Pouladi, D. Khatiwada, V. Selvamanickam, and J. H. Ryou, Sens. Actuators, A 305, 111940 (2020).

${ }^{5}$ H. S. Alpert, C. A. Chapin, K. M. Dowling, S. R. Benbrook, H. Köck, U. Ausserlechner, and D. G. Senesky, Rev. Sci. Instrum. 91, 025003 (2020).

${ }^{6}$ H. Q. Nguyen, T. Dinh, H. A. Moghadam, T. K. Nguyen, T. Nguyen, J. Han, S. Dimitrijev, Y. Zhu, N.-T. Nguyen, and D. V. Dao, IEEE Trans. Electron Devices 68, 1495 (2021).

${ }^{7}$ Z. Hassan, S. S. Ng, G. L. Chew, F. K. Yam, M. J. Abdullah, M. R. Hashim, K. Ibrahim, and M. E. Kordesch, Mater. Sci. Forum 480-481, 531 (2005).

${ }^{8}$ D. Gajula, I. Jahangir, and G. Koley, Micromachines 9, 207 (2018).

${ }^{9}$ O. Ambacher, J. Phys. D. Appl. Phys. 31, 2653 (1998).

${ }^{10}$ O. Ambacher, B. Foutz, J. Smart, J. R. Shealy, N. G. Weimann, K. Chu, M. Murphy, A. J. Sierakowski, W. J. Schaff, L. F. Eastman, R. Dimitrov, A. Mitchell, and M. Stutzmann, J. Appl. Phys. 87, 334 (2000).

${ }^{11}$ J. P. Ibbetson, P. T. Fini, K. D. Ness, S. P. DenBaars, J. S. Speck, and U. K. Mishra, Appl. Phys. Lett. 77, 250 (2000).

${ }^{12}$ O. Ambacher, J. Smart, J. R. Shealy, N. G. Weimann, K. Chu, M. Murphy, W. J. Schaff, L. F. Eastman, R. Dimitrov, L. Wittmer, M. Stutzmann, W. Rieger, and J. Hilsenbeck, J. Appl. Phys. 85, 3222 (1999).

${ }^{13}$ V. Cimalla, J. Pezoldt, and O. Ambacher, J. Phys. D. Appl. Phys. 40, 6386 (2007).

${ }^{14}$ T. Zimmermann, M. Neuburger, P. Benkart, F. J. Hernández-Guillén, C. Pietzka, M. Kunze, I. Daumiller, A. Dadgar, A. Krost, and E. Kohn, IEEE Electron Device Lett. 27, 309 (2006).

${ }^{15} \mathrm{X}$. Wang, R. Yu, C. Jiang, W. Hu, W. Wu, Y. Ding, W. Peng, S. Li, and Z. L. Wang, Adv. Mater. 28, 7234 (2016).

${ }^{16}$ H. Q. Nguyen, H. A. Moghadam, T. Dinh, H. P. Phan, T. K. Nguyen, J. Han, S. Dimitrijev, N. T. Nguyen, and D. V. Dao, Mater. Lett. 244, 66 (2019).

${ }^{17}$ B. S. Kang, J. Kim, S. Jang, F. Ren, J. W. Johnson, R. J. Therrien, P. Rajagopal, J. C. Roberts, E. L. Piner, K. J. Linthicum, S. N. G. Chu, K. Baik, B. P. Gila, C. R. Abernathy, and S. J. Pearton, Appl. Phys. Lett. 86, 253502 (2005).

${ }^{18}$ B. S. Kang, S. Kim, F. Ren, J. W. Johnson, R. J. Therrien, P. Rajagopal, J. C. Roberts, E. L. Piner, K. J. Linthicum, S. N. G. Chu, K. Baik, B. P. Gila, C. R. Abernathy, and S. J. Pearton, Appl. Phys. Lett. 85, 2962 (2004).

${ }^{19}$ B. S. Kang, S. Kim, J. Kim, F. Ren, K. Baik, S. J. Pearton, B. P. Gila, C. R. Abernathy, C. C. Pan, G. T. Chen, J. I. Chyi, V. Chandrasekaran, M. Sheplak, T. Nishida, and S. N. G. Chu, Appl. Phys. Lett. 83, 4845 (2003).

${ }^{20}$ B. S. Kang, S. Kim, J. Kim, R. Mehandru, F. Ren, K. Baik, S. J. Pearton, B. P. Gila, C. R. Abernathy, C. C. Pan, G. T. Chen, J. I. Chyi, V. Chandrasekaran, M. Sheplak, T. Nishida, and S. N. G. Chu, Phys. Status Solidi C 2, 2684 (2005).

${ }^{21}$ Y. Liu, P. P. Ruden, J. Xie, H. Morkoç, and K. A. Son, Appl. Phys. Lett. 88, 013505 (2006).

${ }^{22}$ M. Eickhoff, O. Ambacher, G. Krötz, and M. Stutzmann, J. Appl. Phys. 90 3383 (2001).

${ }^{23}$ T. Lalinský, P. Hudek, G. Vanko, J. Dzuba, V. Kuti, R. Srnánek, P. Choleva, M. Vallo, M. Drík, L. Matay, and I. Kosti, Microelectron. Eng. 98, 578 (2012).

${ }^{24}$ B. Li, X. Tang, H. Li, H. A. Moghadam, Z. Zhang, J. Han, N. T. Nguyen, S. Dimitrijev, and J. Wang, Appl. Phys. Express 12, 064001 (2019).

${ }^{25}$ W. Saito, Y. Takada, M. Kuraguchi, K. Tsuda, and I. Omura, IEEE Trans. Electron Devices 53, 356 (2006).

${ }^{26}$ Y. Cai, Y. Zhou, K. M. Lau, and K. J. Chen, IEEE Trans. Electron Devices 53, 2207 (2006).

${ }^{27}$ Y. Uemoto, M. Hikita, H. Ueno, H. Matsuo, H. Ishida, M. Yanagihara, T. Ueda, T. Tanaka, and D. Ueda, IEEE Trans. Electron Devices 54, 3393 (2007).

${ }^{28}$ F. Roccaforte, G. Greco, P. Fiorenza, and F. Iucolano, Materials 12, 1599 (2019).

${ }^{29}$ S. J. Pearton, B. S. Kang, S. Kim, F. Ren, B. P. Gila, C. R. Abernathy, J. Lin, and S. N. G. Chu, J. Phys.: Condens. Matter 16, R961 (2004).

${ }^{30} \mathrm{O}$. Yilmazoglu, K. Mutamba, D. Pavlidis, and M. R. Mbarga, IEICE Trans. Electron. E89-C, 1037 (2006). 
${ }^{31}$ C. A. Chapin, R. A. Miller, K. M. Dowling, R. Chen, and D. G. Senesky, Sens. Actuators, A 263, 216 (2017)

${ }^{32}$ X. Tan, Y. J. Lv, X. Y. Zhou, Y. G. Wang, X. B. Song, G. D. Gu, P. F. Ji, X. L. Yang, B. Shen, Z. H. Feng, and S. J. Cai, AIP Adv. 8, 085202 (2018).

${ }^{33}$ E. D. le Boulbar, M. J. Edwards, S. Vittoz, G. Vanko, K. Brinkfeldt, L. Rufer, P. Johander, T. Lalinský, C. R. Bowen, and D. W. E. Allsopp, Sens. Actuators, A 194, 247 (2013).

${ }^{34}$ A. S. Yalamarthy and D. G. Senesky, Semicond. Sci. Technol. 31, 035024 (2016).

${ }^{35}$ G. Crystal, W. Soluch, L. S. Member, E. Brzozowski, and M. Lysakowska, IEEE Trans. Ultrason., Ferroelectr., Freq. Control 58, 2469 (2011).

${ }^{36}$ F. Bernardini, V. Fiorentini, and D. Vanderbilt, Phys. Rev. B 56, R10024 (1997).

${ }^{37}$ A.-B. Chen, A. Sher, and W. T. Yost, The Mechanical Properties of Semiconductors, edited by K. T. Faber and K. J. Malloy (Elsevier, 1992), pp. 1-77.
${ }^{38}$ M. Chu, Y. Sun, U. Aghoram, and S. E. Thompson, Annu. Rev. Mater. Res. 39, 203 (2009).

${ }^{39}$ S. Adachi, Properties of Semiconductor Alloys: Group-IV, III-V and II-VI Semiconductors (Wiley, New York, 2009).

${ }^{40}$ U. Rössler, Solid State Commun. 49, 943 (1984).

${ }^{41}$ B. Jogai, Phys. Rev. B 57, 2382 (1998).

${ }^{42}$ S. Postorino, D. Grassano, M. D'Alessandro, A. Pianetti, O. Pulci, and M. Palummo, Nanomater. Nanotechnol. 10, 184798042090256 (2020).

${ }^{43}$ M. Chu, A. D. Koehler, A. Gupta, T. Nishida, and S. E. Thompson, J. Appl. Phys. 108, 104502 (2010).

${ }^{44}$ Y. Sun, G. Sun, S. Parthasarathy, and S. E. Thompson, Mater. Sci. Eng., B 135, 179 (2006). 